\title{
Desempeño del número de empleos en tiempos de COVID-19 en dos estados mexicanos con distinta vocación económica
}

\author{
LAgunAs Puls, SERGIO \\ Universidad del Caribe (México) \\ Correo electrónico: slagunas@ucaribe.edu.mx \\ DOMÍNGUEZ ESTRADA, JOSÉ FRANCISCO \\ Universidad del Caribe (México) \\ Correo electrónico: fdominguez@ucaribe.edu.mx \\ OROPEZA TAgLE, Miguel ÁNGEL \\ Universidad Autónoma de Aguascalientes (México) \\ Correo electrónico: maoropez@correo.uaa.mx
}

\begin{abstract}
RESUMEN
El objetivo de este artículo es medir el desempeño del trabajo formal en dos estados de México con vocación económica distinta: Quintana Roo cuya actividad principal es el turismo y Aguascalientes con actividad principalmente industrial. El trabajo se inicia presentando el contexto laboral en ambos estados y posteriormente se describe la revisión de la literatura relacionada al desempeño de los trabajos formales en México y Latinoamérica. Con los datos oficiales de los trabajadores afiliados al IMSS de 1998 a 2019 se identifica el mejor ajuste; con los parámetros estadísticos obtenidos se analiza el comportamiento mensual del primer cuatrimestre del año 2020 con metodología clásica contrastada con metaanálisis. Los resultados permiten concluir que en periodos previos y durante el distanciamiento social, el número de afiliados al IMSS no solo se mantuvo en niveles convencionales, sino que en varios meses se incrementaron los trabajadores inscritos.
\end{abstract}

Palabras clave: trabajo formal, desempeño laboral, COVID-19.

Clasificación JEL: C13; C16; C46.

MSC2010: 62F10; 62F25; 33E20. 


\title{
Performance of the number of jobs in times of COVID- 19 in two Mexican states with different economic vocation
}

\begin{abstract}
The objective of this paper is to measure the performance of formal work in two states of Mexico with different economic vocation: Quintana Roo whose main activity is tourism and Aguascalientes with mainly industrial activity. The work begins by presenting the work context in both states, and later describes the review of literature related to the performance of formal work in Mexico and Latin America. With the official data of the workers affiliated to the IMSS from 1998 to 2019, the best adjustment is identified, with the statistical parameters obtained; the monthly behavior of the first quarter of 2020 is analyzed with classical methodology contrasted with meta-analysis. The results allow us to conclude that in previous periods and during social distancing, the number of IMSS members not only remained at conventional levels, but that in several months the number of registered workers increased.
\end{abstract}

Keywords: labor, formal work, COVID-19.

JEL classification: C13; C16; C46.

MSC2010: 62F10; 62F25; 33E20.

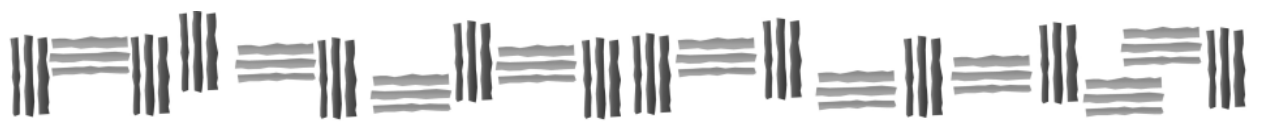




\section{Introducción}

Es de señalar la situación del empleo en México, correspondiente al primer trimestre de 2020, donde se registran 57,3 millones de personas en la categoría conocida como Población Económicamente Activa (PEA), y de esta cantidad, la Población Ocupada (POC) alcanzó 55,3 millones de personas, de los que 33,5 millones fueron hombres y 21,9 millones mujeres. Para la entidad de Quintana Roo la PEA representó el $65,2 \%$ de la población total, cantidad de la que el 97,1\% se encuentra ocupada, mientras que en Aguascalientes la PEA representó el 59,5\% de la población, de la que el 96,7\% se encuentra ocupada (INEGI, 2020). Para el caso de Aguascalientes, no existen datos del total del número de personas de la población económicamente activa, solo existen datos en porcentajes.

La dinámica de la PEA como de la POC puede analizarse desde distintos enfoques, siendo uno de estos, de interés particular para el presente artículo: el número de trabajadores afiliados al Instituto Mexicano del Seguro Social (IMSS), es decir, el número de personas ocupadas que gozan de un trabajo formal, que genera aportaciones de seguridad social, vivienda y recaudación fiscal de cuya base es el salario (impuesto sobre nóminas y conceptos asimilados; impuesto sobre la renta). Con esta información se establecerán los patrones estadísticos de periodos mensuales, desde 1998 y hasta 2019, de tal forma que se pueda evaluar el impacto de la pandemia por COVID-19 en México, contrastando los resultados para estados con vocaciones económicas distintas: Quintana Roo cuya vocación recae en los servicios turísticos, construcción y servicios relacionados y Aguascalientes cuya vocación económica es industrial (IMSS, 2020).

Esto se pudiera explicar considerando que en ambos Estados, se encuentran grandes conglomerados empresariales: en Aguascalientes un buen número de grandes empresas industriales del sector automotriz y de autopartes, mientras que en Quintana Roo está constituido por grandes empresas de servicios, que optaron por no despedir a los empleados, sino aplicar tratamiento de descansar a empleados con disminución de salarios, jornales y aplicar esquemas de teletrabajo (Home office).

\section{Marco referencial}

De acuerdo con la Organización Internacional del Trabajo, millones de personas durante la pandemia por COVID-19 tuvieron, en el mejor de los casos, la necesidad de quedarse en casa e iniciar sus actividades laborales a distancia. No obstante, en millones de casos las personas tuvieron que sopesar reducción en las contraprestaciones o, en casos aún más graves, la pérdida de la relación laboral con el consecuente impacto en las finanzas personales, llegándose a estimar que la afectación del empleo en México alcanzaría los 24 millones de personas, lo que representaría más del $40 \%$ del empleo formal del país (Organización Internacional del Trabajo, 2020).

Respecto al impacto en el número de puestos de trabajo, se ha considerado que puede ser aminorado siempre que exista compromiso del gobierno federal y de los estados, estímulos a la economía mediante estímulos fiscales que fortalezcan las fuentes de trabajo y, en consecuencia, puedan evitarse la reducción de algunos empleos, sabiendo que, algunos de los sectores en los que se pueden esperar mayores consecuencias es en turismo, como lo que acontece en Quintana Roo, pero también en el de manufacturas que tiene presencia en el estado de Aguascalientes (Aguilar, 2020).

En este mismo sentido, se ha observado que entre las entidades del país que presentaron grave reducción en las plazas laborales son aquellas en cuya actividad económica se encuentran los servicios turísticos, expresando que tan solo en dos meses, abril y mayo del 2020, Quintana Roo perdió más de noventa mil puestos de trabajo, tan solo por debajo de la pérdida de empleo que registró la Ciudad de México en estos mismos períodos (de Jesús \& Murillo, 2020).

Por su parte, la Organización para la Cooperación y el Desarrollo Económicos (OCDE) indica que el empleo formal es el que más impacto tiene en el crecimiento económico y en la reducción de la 
pobreza, en donde evidentemente, los empleados formales se encuentran bajo un régimen tributario y con sus contribuciones fiscales otorgan al Estado un ingreso mayor que el de los trabajadores informales, debido a que los últimos solamente producen bienes y servicios más necesariamente contribuyen con el ingreso público. Por lo tanto, la reproducción de oficios remuneradores es esencial para avivar el crecimiento, comprimir la escasez y acrecentar la conexión social (OCDE, 2020).

En México, el empleo formal se mide principalmente a través del número de trabajadores registrados ante el IMSS, siendo la institución encargada de brindar seguridad social a los trabajadores registrados; por otro lado, el empleo informal describe a la actividad laboral independiente, los vendedores ambulantes, las trabajadoras de servicio doméstico, o bien, todos aquellos obreros que no tienen una relación laboral reconocida frente al Estado. Negrete (2011) declara que la tasa de desempleo no es el mejor indicador para medir la dinámica del mercado laboral de un país, es decir, la actividad económica mexicana no se ajusta al aumento de desempleos, sino por la disminución de salarios, el aumento del subempleo, el empleo informal y las malas condiciones de trabajo a las que están sujetas millones de trabajadores.

Hay que considerar que el acceso a un empleo de calidad en cualquier país del mundo, es el medio más eficaz para erradicar la penuria, debido a que, la ampliación de los ingresos profesionales es responsable de alrededor del $40 \%$ de la disminución de la pobreza en la última década a nivel internacional. La mayoría de los países en desarrollo como México afrontan, en distintos grados, tres desafíos primordiales: a) crear más empleos, b) mejorar la calidad de los empleos y c) conectar a las personas con las fuentes de empleo; no toda la mano de obra disfruta de las mismas oportunidades laborales (la juventud, las mujeres y los más necesitados son los más menospreciados en el mercado laboral). Se debe afirmar que la clase obrera tiene la capacidad que se necesita para descartar todas las formas de segregación al empleo (Banco Mundial, 2020).

Quizá por el interés general es por lo que existen programas de fomento, siendo aquellos que efectúa el Estado con el objeto de generar los ambientes adecuados para el impulso e incentivación del empleo entre la población. Por medio de los programas se explora la forma de solucionar los problemas que sufren tanto la oferta, como la demanda para encontrarse en el mercado laboral, de quienes buscan empleo, señalando la falta de promoción, la carencia de conocimientos, destrezas laborales, la falta de recursos para buscar y colocarse en un puesto de trabajo, o iniciar una actividad por cuenta propia, en el caso de la población joven la falta de experiencia laboral, o los grupos de población de adultos mayores y personas con alguna discapacidad.

\section{Retos y esfuerzos para 2020}

En México, el gobierno federal y cada Estado de la República contemplan en sus planes de desarrollo programas de fomento al empleo como: el Programa de Apoyo al Empleo, el Programa de Apoyo a la Capacitación en el Trabajo, el Fomento a la Productividad, el Programa de Fomento al Trabajo Digno, entre otros. En el ámbito de los efectos del COVID-19 sobre la economía, Rivero (2020) argumentó que existen proyecciones que prevén una crisis de empleo en México, es por ello que, el Presidente de la República, Andrés Manuel López Obrador, mediante decreto afirmó que se irían dando a conocer los ajustes al plan de reactivación económica a partir del 23 de abril de 2020 (Presidencia de la República, 2020), mencionando su deseo para luchar por la creación de dos millones de empleos buscando la protección de los más pobres, pero reconociendo que durante los primeros meses del año 2020 se perdieron 148.845 puestos de trabajo, 21.263 trabajos al día, especificando que seis entidades concentraron el 56\% del total de las separaciones laborales: Quintana Roo 63.847, Ciudad de México 55.591, Nuevo León 23.465, Jalisco 21.535, Estado de México 16.036 y Tamaulipas 12.652.

También la publicación del Decreto mencionado anteriormente señalaba compañías que habían cesado a trabajadores infundadamente, sin pago de sueldos y salarios, en donde el 47,5\% de las ocupaciones perdidas se efectuaron en medianas empresas; entre las acciones más afectadas se hallarían 
la industria de la transformación (automotriz, maquinaria y equipo eléctrico, entre otros), perjudicando directamente al estado de Aguascalientes.

Por otra parte, la industria de la construcción y turismo se vieron afectadas en todo el país, pero especialmente Quintana Roo y Baja California Sur, ocasionando erosión en otras actividades como los servicios pedagógicos, bancarios, industriales, edificación, inmobiliarios y marítimos; esto se puede notar en contraste, con servicios menos afectados como los médicos, alimentos, tecnologías, comunicación, comercio y agricultura.

Entre las medidas indicadas por las entidades federativas ante la emergencia, el gobernador de Quintana Roo, Carlos Joaquín González, declaró que el estado trabajó en medidas de prevención, que se intensificaron el 14 de marzo. Entre ellas destacaron: medidas sanitarias (control de higiene, aislamiento social, test diarios, permanencia en casas, no asistir a lugares masivos); medidas económicas (suspensión de horarios para establecimientos que expendan bebidas alcohólicas, y cierre de establecimientos con concurrencia masiva); estímulos fiscales (diferimiento de pago de los impuestos, condonación del $20 \%$ para pago en tiempo y forma de los impuestos, condonación de multas e intereses moratorios, suspensión de actos de fiscalización estatales, ampliación para pago de tenencias y placas vehiculares, registro público); apoyo con abastecimiento de agua (servicio garantizado sin cortes ni sanciones); micro, pequeñas y medianas empresas (programa estatal de financiación al emprendedor, a la actividad artesanal, impulso a las empresas); servicios en línea (pagos en línea por cierre parcial de oficinas públicas); apoyo de médico en tu casa (a través del 911 con médicos de todas las especialidades, psicólogos, gratuitas); apoyo con créditos (productos hechos en quintana roo); así como también de apoyos con la energía eléctrica y gas LP, apoyando desde lo social a la gente y preparando los destinos turísticos para la recuperación con campañas de promoción a mercados regionales para reactivar la demanda como meta de turismo para este año (Gobierno de Quintana Roo, 2020).

En el estado de Aguascalientes, el Gobernador, Martín Orozco Sandoval, presentó el Programa "Todos por Aguascalientes", a finales de marzo, con una Bolsa de 1.800 millones de pesos, con el objetivo de aminorar el impacto económico del COVID-19, protegiendo las finanzas estatales y al empresariado, destacando: financiamiento y subsidio a MIPYMES, impulso al consumo local (ayudando a 56.706 personas y 500 empresas), apoyo al campo, inversión de obra pública (generando 5.000 empleos y salvaguardando a 138 empresas), incentivos empresariales, apoyo alimenticio para grupos vulnerables, fortalecimiento de la economía familiar, fondo especial para empresarios y sus trabajadores (dirigido a apoyar trabajadores que se han quedado sin empleo), apoyo a micronegocios (para Mujer emprendedora, Talento Joven, Fondo Incluyente y Fondo Fortalece), respaldo a comercios locales (a través de envíos a domicilio, con el fin de que los comercios sigan trabajando y abasteciendo a la sociedad) y capacitación en línea para incrementar las ventas online (Patiño \& Cruz, 2020).

De acuerdo a lo anterior, algunos estados de México, como el caso de Aguascalientes y Quintana Roo, han procurado apuntalar sus economías, con distintos elementos. Quizá debido a la vocación económica particular, por tales circunstancias se vuelve interesante y a la vez necesario, realizar análisis comparado del nivel de cambios en el empleo formal en estos dos estados, durante los meses del primer cuatrimestre del año 2020, conociendo cuáles han sido los efectos causados por la pandemia de COVID19. Por lo anterior, en el siguiente apartado se indicará la metodología aplicada, alineada con el objetivo general y la principal incógnita a resolver.

\section{Metodología y datos}

El objetivo general del presente artículo es establecer el nivel de cambios en el número de trabajadores afiliados al Instituto Mexicano del Seguro Social (IMSS), durante la epidemia por COVID-19. Se considera que los primeros dos meses de 2020 en que ya estaba declarada contingencia en otros países, 
pudo incidir indirectamente en la dinámica laboral y posteriormente, de manera directa, el aviso oficial para distanciamiento social en México a partir de marzo y durante el mes de abril.

La principal incógnita para responder será la siguiente: ¿Cuáles fueron los cambios y la magnitud en el número de trabajadores afiliados al IMSS durante los meses de enero, febrero, marzo y abril 2020 ?

La variable de interés es el número de trabajadores afiliados al IMSS, con amplitud de datos de 1998 a 2019, en agrupaciones mensuales (enero, febrero, marzo y abril) tanto para el estado de Aguascalientes como Quintana Roo.

Tabla 1. Trabajadores afiliados al IMSS en el estado de Aguascalientes, 1998-2019

\begin{tabular}{|c|c|c|c|c|}
\hline Año & Enero & Febrero & Marzo & Abril \\
\hline 1998 & 145.731 & 148.066 & 150.723 & 153.166 \\
1999 & 156.020 & 158.006 & 162.293 & 162.514 \\
2000 & 172.842 & 176.183 & 178.610 & 179.954 \\
2001 & 182.726 & 184.084 & 184.271 & 185.603 \\
2002 & 176.427 & 176.821 & 176.646 & 181.271 \\
2003 & 180.198 & 180.560 & 180.962 & 182.889 \\
2004 & 178.088 & 177.737 & 179.476 & 179.840 \\
2005 & 183.521 & 186.458 & 186.735 & 187.162 \\
2006 & 188.115 & 189.904 & 191.779 & 193.274 \\
2007 & 203.834 & 205.371 & 206.779 & 208.786 \\
2008 & 208.017 & 206.724 & 207.545 & 209.679 \\
2009 & 199.151 & 198.237 & 197.510 & 197.451 \\
2010 & 200.746 & 202.236 & 204.779 & 207.188 \\
2011 & 205.433 & 207.327 & 209.918 & 210.529 \\
2012 & 214.683 & 216.472 & 218.836 & 220.053 \\
2013 & 230.266 & 232.142 & 233.925 & 235.947 \\
2014 & 242.758 & 245.654 & 247.663 & 249.575 \\
2015 & 257.555 & 260.487 & 262.850 & 264.182 \\
2016 & 274.146 & 278.093 & 280.339 & 282.761 \\
2017 & 290.744 & 293.966 & 297.378 & 298.118 \\
2018 & 307.118 & 310.545 & 312.853 & 316.044 \\
2019 & 323.981 & 325.728 & 326.846 & 327.345 \\
\hline
\end{tabular}

Fuente: Elaboración propia con datos del Instituto Mexicano de Seguro Social (IMSS, 2020).

Tabla 2. Trabajadores afiliados al IMSS en el estado de Quintana Roo. 1998-2019.

\begin{tabular}{|c|c|c|c|c|}
\hline Año & Enero & Febrero & Marzo & Abril \\
\hline 1998 & 131.110 & 133.783 & 133.815 & 136.513 \\
1999 & 152.060 & 157.129 & 155.282 & 155.865 \\
2000 & 155.477 & 157.957 & 162.379 & 162.436 \\
2001 & 171.640 & 175.119 & 178.218 & 177.155 \\
2002 & 166.999 & 170.181 & 173.212 & 172.640 \\
2003 & 179.926 & 181.365 & 183.052 & 183.977 \\
2004 & 192.318 & 195.447 & 199.145 & 200.207 \\
2005 & 215.652 & 219.483 & 220.441 & 224.263 \\
2006 & 222.711 & 227.077 & 231.409 & 231.902 \\
2007 & 250.485 & 254.008 & 256.650 & 258.126 \\
2008 & 265.923 & 270.821 & 272.556 & 274.433 \\
2009 & 268.545 & 269.006 & 270.648 & 268.492 \\
2010 & 258.567 & 260.704 & 265.696 & 260.789 \\
2011 & 269.063 & 271.697 & 272.369 & 272.525 \\
2012 & 272.756 & 273.948 & 276.897 & 275.269
\end{tabular}




\begin{tabular}{lllll}
2013 & 285.544 & 288.951 & 291.352 & 290.836 \\
2014 & 299.706 & 303.767 & 306.989 & 308.393 \\
2015 & 320.889 & 323.040 & 326.901 & 328.451 \\
2016 & 348.633 & 353.634 & 357.584 & 359.875 \\
2017 & 382.352 & 388.977 & 393.536 & 394.646 \\
2018 & 425.496 & 431.285 & 434.918 & 441.383 \\
2019 & 458.585 & 459.222 & 458.246 & 463.068 \\
\hline
\end{tabular}

Fuente: Elaboración propia con datos del Instituto Mexicano de Seguro Social (IMSS, 2020).

Con la información contenida en las Tablas 1 y 2 , se realizaron pruebas de ajuste para distribución normal y lognormal, siendo el parámetro de decisión el estadístico Anderson-Darling (Grace \& Wood, 2012; Shin et al., 2012), representado por $A^{2}$ con nivel crítico de 0,752 es decir, cuanto menor sea el resultado para $A^{2}$ se entenderá un mejor ajuste.

$$
A^{2}=-n-\left(\frac{1}{n}\right) \sum\left(2_{i}-1\right)\left\{\ln F\left(Y_{i}\right)+\ln \left(1-F\left(Y_{n+1-i}\right)\right)\right\}
$$

donde:

$$
\begin{aligned}
& n=\text { número de datos de afiliados al IMSS } \\
& \text { ln = logaritmo natural } \\
& F=\text { distribución con función acumulativa }
\end{aligned}
$$

La función con mejor ajuste se utilizará para calcular parámetros probabilísticos de dos extremos con significancia de $\alpha / 2=0,025$. Las curvas de distribución normal se obtendrán a partir de la siguiente función:

$$
f_{\text {normal }}(x ; \mu, \sigma)=\frac{e^{-\frac{(x-\mu)^{2}}{\sigma^{2}}}}{|\sigma| \sqrt{2 \pi}} ; x>0
$$

Por lo tanto, el área bajo la curva para establecer los límites al nivel de significación estará dada por la siguiente integral definida:

$$
\int_{\theta}^{\rho} f_{\text {normal }}(x ; \mu, \sigma) d x=\int_{\theta}^{\rho} \frac{e^{-\frac{(x-\mu)^{2}}{\sigma^{2}}}}{|\sigma| \sqrt{2 \pi}} ; x>0
$$

donde:

$\mu=$ media del número de afiliados al IMSS

$\sigma=$ desviación estándar del número de afiliados al IMSS

$\pi=P i \sim 3,141$

$x=$ número de afiliados en un período mensual a evaluar

En cuanto a la distribución lognormal, la función de densidad para representar las curvas estará dada por la siguiente función (Dey \& Kundu, 2009):

$$
f_{\text {lognormal }}(x ; \eta, \tau)=\frac{1}{\sqrt{2 \pi x \eta}} e^{-\frac{1}{2}\left(\frac{(\ln (x)-\ln (\tau))^{2}}{\eta^{2}}\right)} ; x>0
$$


Por lo tanto, el área bajo la curva con límite a los valores que expresen el nivel de significación de $\alpha / 2=0,05$ estarán comprendida en la integral definida siguiente:

$$
\int_{\theta}^{\rho} f_{\text {lognormal }}(x ; \eta, \tau) d x=\int_{\theta}^{\rho} \frac{1}{\sqrt{2 \pi x \eta}} e^{-\frac{1}{2}\left(\frac{(\ln (x)-\ln (\tau))^{2}}{\eta^{2}}\right)} d x ; x>0
$$

donde:

ln = logaritmo natural

$\eta=$ parámetro de forma en el número de afiliados al IMSS, análogo a $\mu$

$\tau=$ parámetro de escala en el número de afiliados al IMSS, análogo de $\sigma$

$\pi=P i \sim 3,141$

$x=$ número de afiliados en un período mensual a evaluar

Después de elegir la distribución para cada serie (mes/estado del país), se realizan evaluaciones para el número de trabajadores registrados, representando los valores en gráficas de distribución normal, ubicando en este cada valor, calificando si pueden considerarse cambios significativos bajo criterios clásicos.

Los resultados anteriores se contrastan con el estadístico $d$ de Cohen (Cumming, 2013):

$$
d=\frac{(x-\lambda)}{\zeta}
$$

donde:

$d=$ delta de Cohen

$x=$ trabajadores afiliados al IMSS en un período a evaluar

$\lambda=$ forma o media del número de afiliados al IMSS

$\zeta=$ escala o desviación estándar del número de afiliados al IMSS

Estableciendo criterios adecuados para tres categorías de cambios:

$$
\begin{aligned}
& \text { cambio menor } \rightarrow 0,00 \leq d \leq 1 \\
& \text { cambio medio } \rightarrow 1<d \leq 2 \\
& \text { gran cambio, cuando } d>2
\end{aligned}
$$

\section{Resultados}

Respecto a las pruebas de ajuste, es importante destacar que se realizaron por grupos de meses, es decir, enero 1998, enero $1999 \ldots$ enero 2019; febrero 1998, febrero $1999 \ldots$ febrero 2019 ; marzo 1998, marzo 1999... marzo 2019; abril 1998, abril 1999... abril 2019.

De acuerdo a la información de la Tabla 3, en todos los casos resultó mejor ajuste a distribución lognormal, por lo tanto, serán los parámetros empleados para la medición de cambios. 
Tabla 3. Pruebas de ajuste $A^{2}$ para los meses de enero a marzo 2020.

\begin{tabular}{l|ccc|c|}
\hline \multirow{2}{*}{ Meses } & \multicolumn{2}{|c|}{ Normal } & \multicolumn{2}{c|}{ Lognormal } \\
\cline { 2 - 5 } & Aguascalientes & Quintana Roo & Aguascalientes & Quintana Roo \\
\hline Enero & 0,813 & 0,381 & 0,476 & 0,242 \\
Febrero & 0,873 & 0,402 & 0,528 & 0,254 \\
Marzo & 0,905 & 0,391 & 0,559 & 0,244 \\
Abril & 0,934 & 0,414 & 0,594 & 0,238 \\
\hline
\end{tabular}

Fuente: Elaboración propia.

En lo referente a los estadísticos para evaluar lo meses de 2020 bajo parámetros lognormales, en la Tabla 4 se presentan los estimados de forma y escala.

Tabla 4. Estadísticos de forma y escala para los meses de enero a abril 2020.

\begin{tabular}{|c|c|c|c|}
\hline Meses de 2020 & Estado & Forma & Escala \\
\hline \multirow{2}{*}{ Enero } & Aguascalientes & 12,25 & 0,2158 \\
\cline { 2 - 4 } & Quintana Roo & 12,41 & 0,3432 \\
\hline \multirow{2}{*}{ Febrero } & Aguascalientes & 12,26 & 0,2160 \\
\cline { 2 - 4 } & Quintana Roo & 12,42 & 0,3393 \\
\hline \multirow{2}{*}{ Marzo } & Aguascalientes & 12,27 & 0,2147 \\
\cline { 2 - 4 } & Quintana Roo & 12,43 & 0,3384 \\
\hline \multirow{2}{*}{ Abril } & Aguascalientes & 12,28 & 0,2133 \\
\cline { 2 - 4 } & Quintana Roo & 12,43 & 0,3388 \\
\hline
\end{tabular}

Fuente: Elaboración propia.

Los cambios se evalúan, en primer lugar, con criterios clásicos, ubicando en la gráfica de distribución si cada cantidad evaluada se encuentra, o no, en el área de la integral definida por los valores entre los cuales se encuentra el $95 \%$ de probabilidad.

Tabla 5. Trabajadores afiliados al IMSS en el primer cuatrimestre de 2020.

\begin{tabular}{|c|c|c|c|c|}
\hline Estados & Enero & Febrero & Marzo & Abril \\
\hline Aguascalientes & 335.280 & 338.559 & 336.383 & 328.581 \\
\hline Quintana Roo & 472.299 & 472.041 & 424.238 & 379.254 \\
\hline
\end{tabular}

Fuente: Elaboración propia.

En las siguientes evaluaciones de los meses enero y febrero, comprenden el período previo al distanciamiento social pero ya con presencia del COVID-19 a nivel mundial. 
Figura 1. Cambio en trabajadores afiliados al IMSS en Aguascalientes: enero 2020.

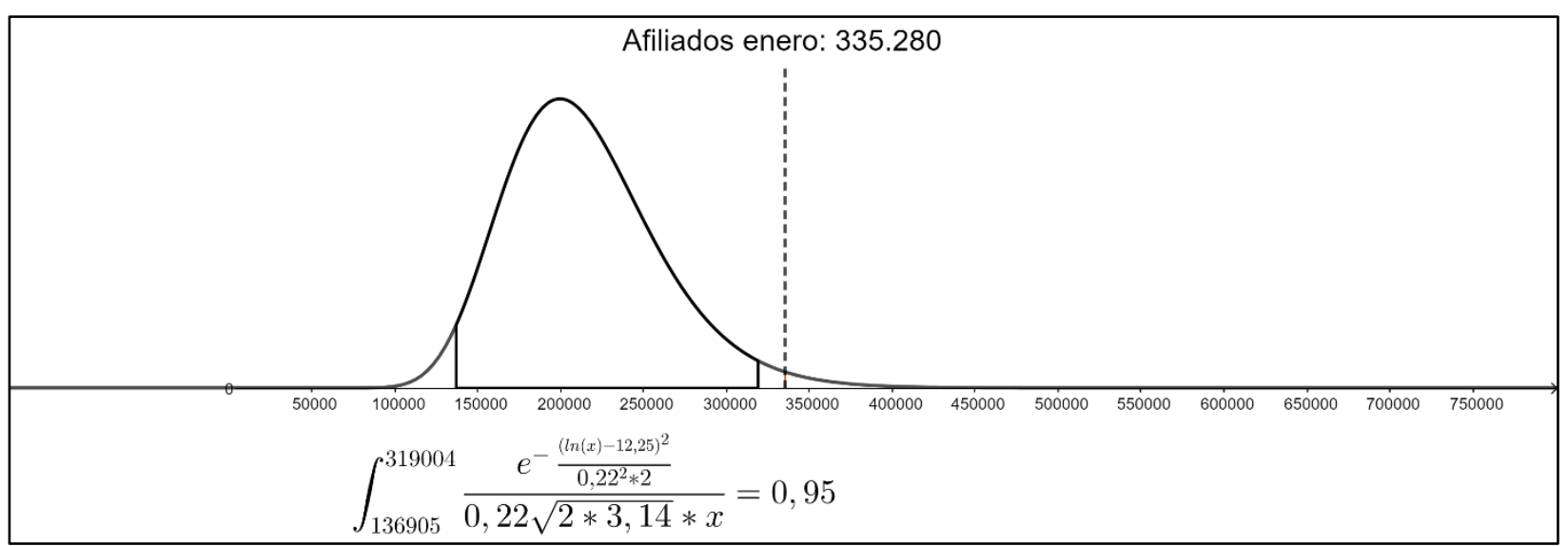

Fuente: Elaboración propia mediante GeoGebra.

Figura 2. Cambio en trabajadores afiliados al IMSS en Quintana Roo: enero 2020.

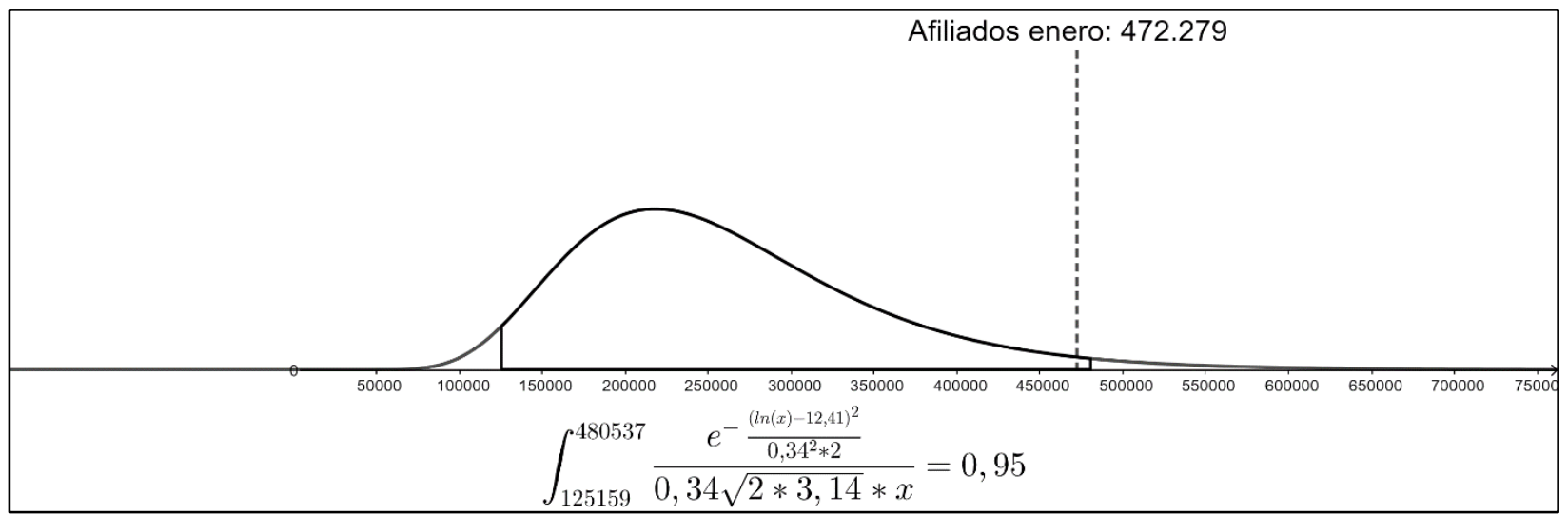

Fuente: Elaboración propia mediante GeoGebra.

El cambio en el número de afiliados para el mes de enero resultó de incremento significativo, fuera del límite normal para el estado de Aguascalientes. No obstante, en el estado de Quintana Roo el valor de esta variable se encontró dentro de lo normal, aunque muy cercano al límite máximo.

En el mes de febrero el estado de Aguascalientes presentó cambio incremental, significativo, respecto a Quintana Roo, el que, al igual que en el mes de enero, sí se presentó incremento, pero sin que pueda llegar a considerarse significativo.

En las siguientes dos evaluaciones, marzo y abril, estaría comprendido el efecto ocasionado por períodos en plenas medidas de distanciamiento, de lo que se esperaría reducción significativa en los trabajadores afiliados. 
Figura 3. Cambio en trabajadores afiliados al IMSS en Aguascalientes: febrero 2020.

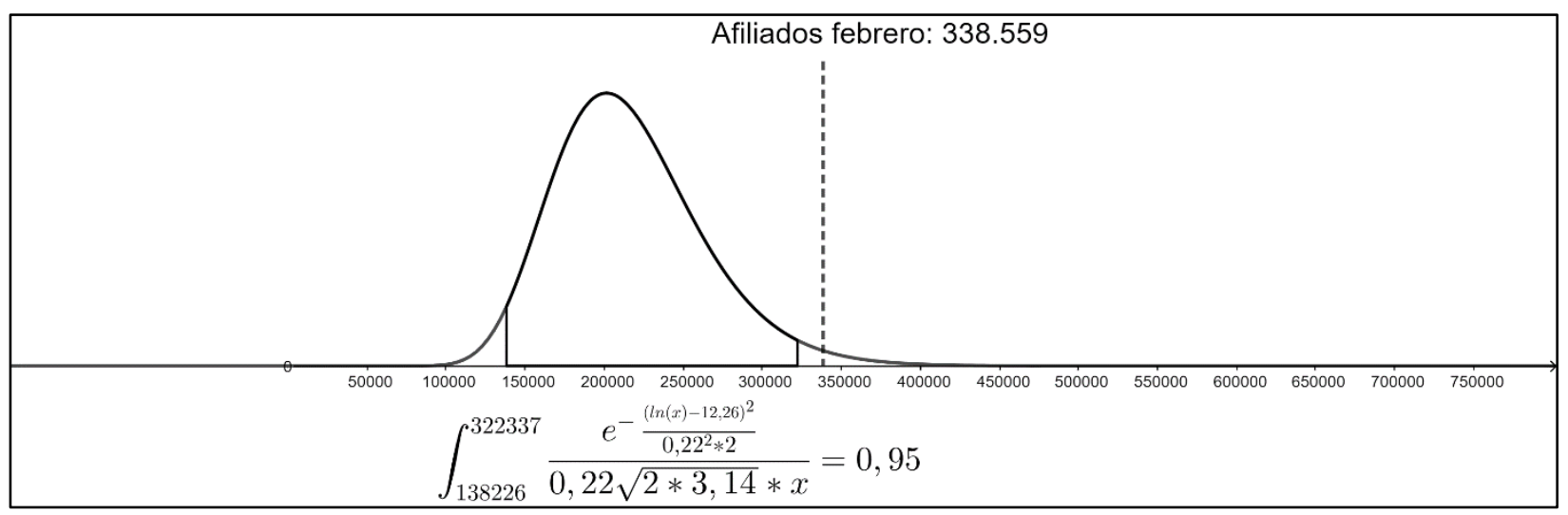

Fuente: Elaboración propia mediante GeoGebra.

Figura 4. Cambio en trabajadores afiliados al IMSS en Quintana Roo: febrero 2020.

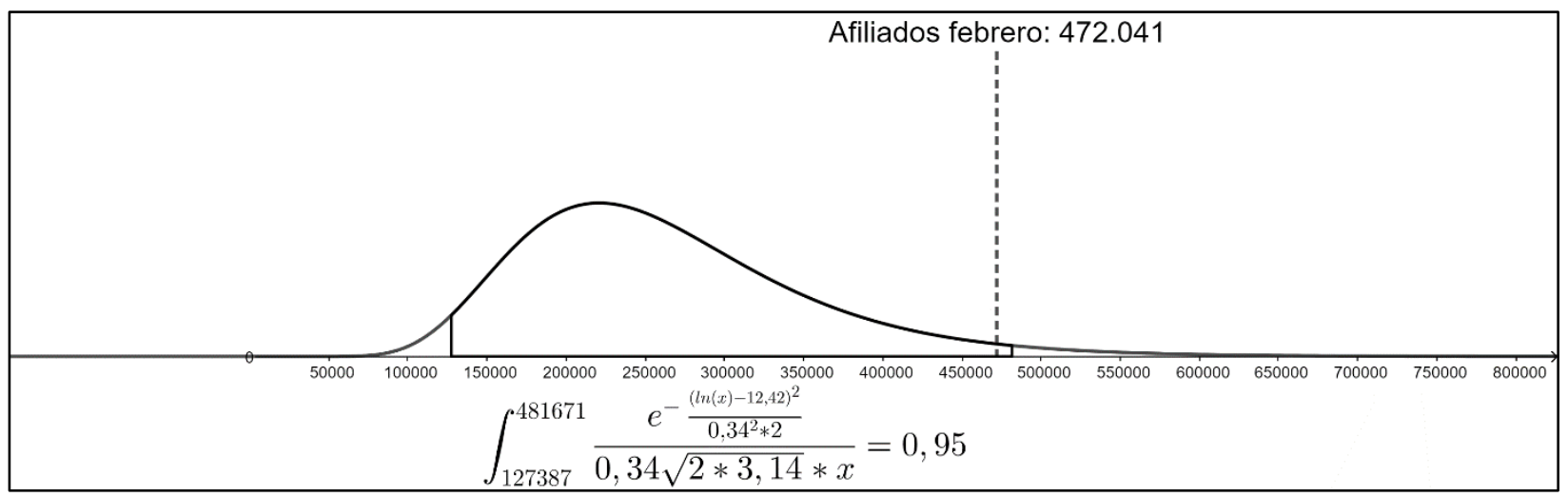

Fuente: Elaboración propia mediante GeoGebra.

Las evaluaciones para el mes de marzo (Figuras 5 y 6) muestran que en Aguascalientes, contrariamente a lo que se podría haber supuesto, el número de trabajadores se incrementó significativamente; sin embargo, en el estado de Quintana Roo no se alcanzó cambio significativo, aunque, como en los meses de enero y febrero, sí se presentó incremento, cercano al límite para considerarse cambio incremental significativo.

Figura 5. Cambio en trabajadores afiliados al IMSS en Aguascalientes: marzo 2020.

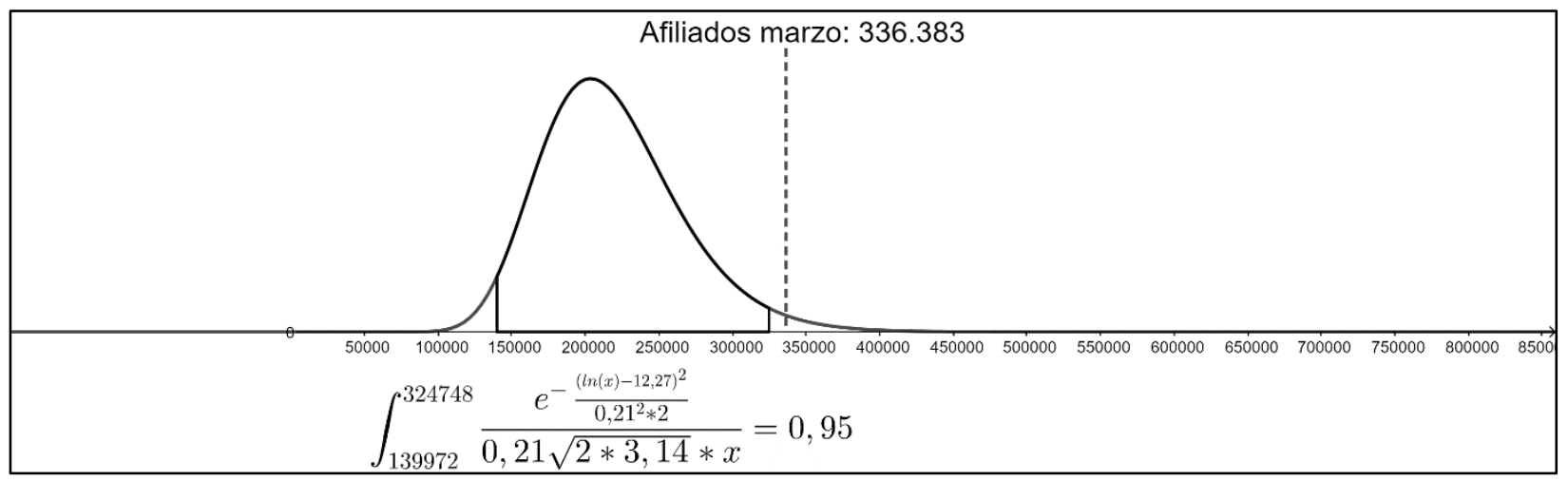

Fuente: Elaboración propia mediante GeoGebra. 
Figura 6. Cambio en trabajadores afiliados al IMSS en Quintana Roo: marzo 2020.

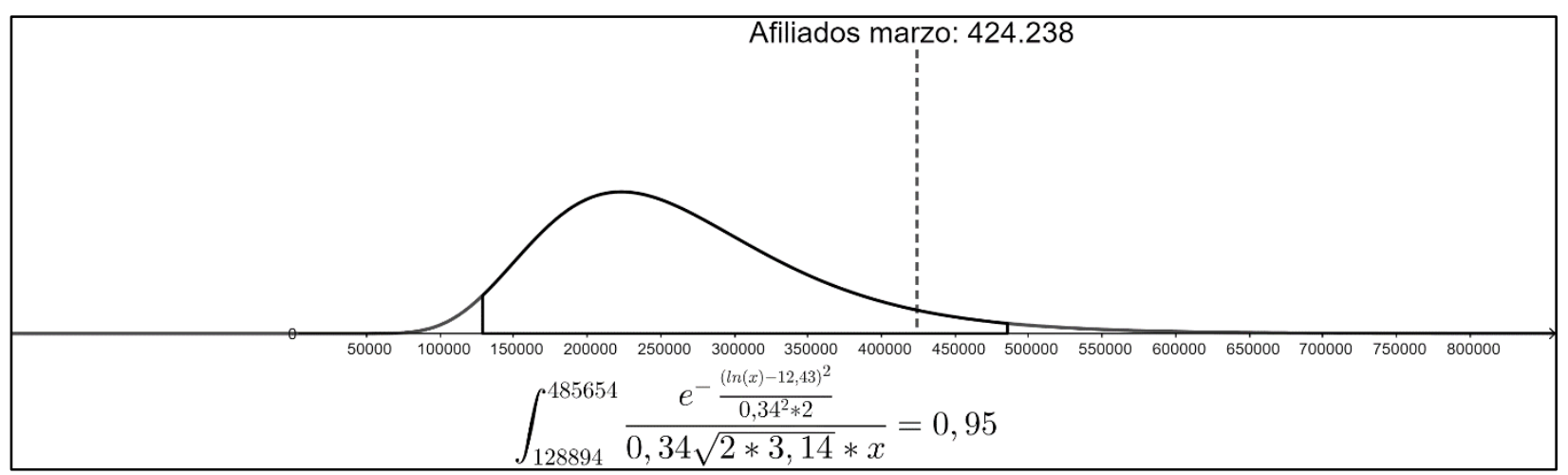

Fuente: Elaboración propia mediante GeoGebra.

Finalmente se presentan los resultados para el mes de abril, período que pudiera denominarse como "en profundo distanciamiento":

Figura 7. Cambio en trabajadores afiliados al IMSS en Aguascalientes: abril 2020.

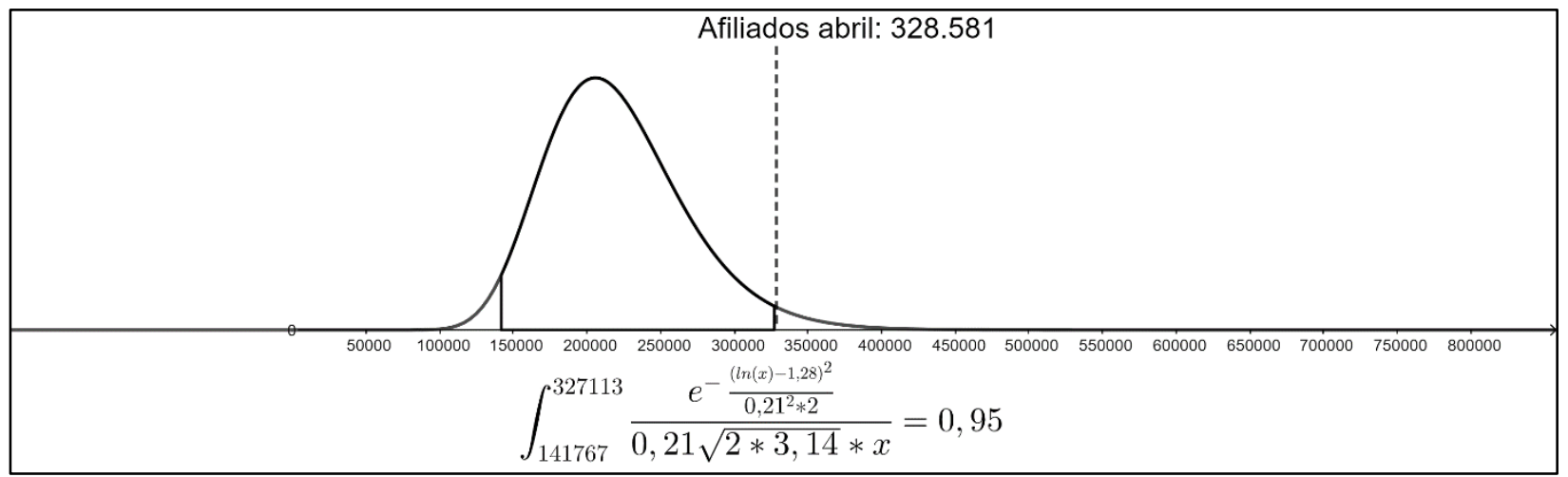

Fuente: Elaboración propia mediante GeoGebra.

Figura 8. Cambio en trabajadores afiliados al IMSS en Quintana Roo: abril 2020.

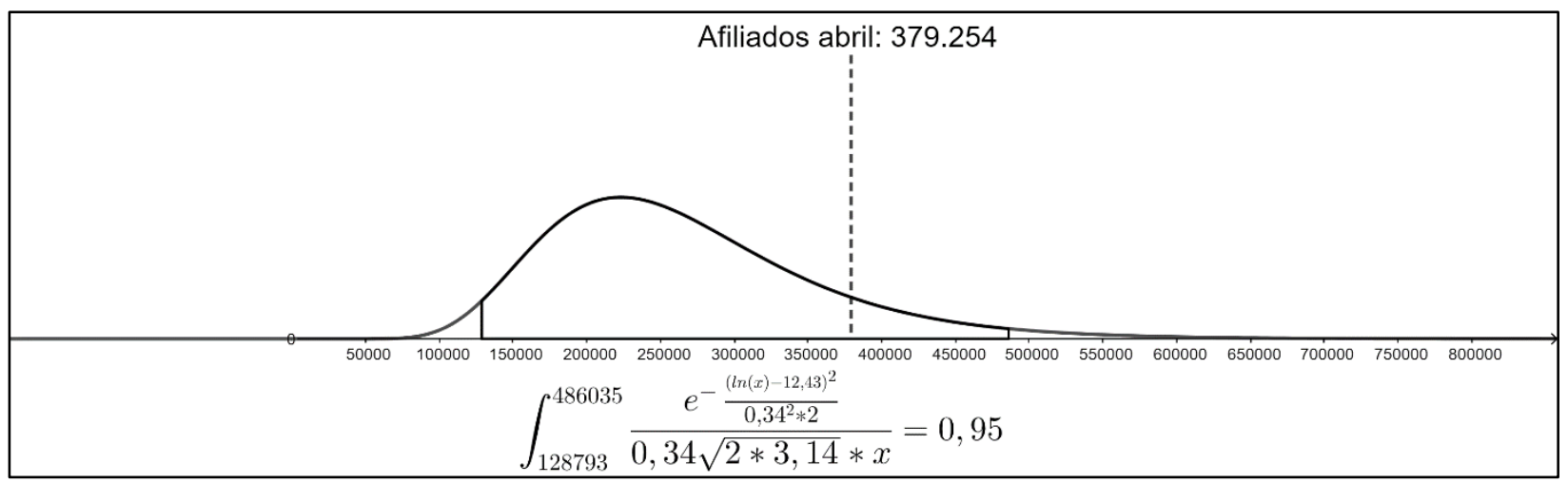

Fuente: Elaboración propia mediante GeoGebra.

Las evaluaciones en el mes de abril permiten apreciar particularidades interesantes. En el primer caso analizado, Aguascalientes, el número de afiliados resultó incremental y significativo, aunque en 
menor medida que los meses anteriores. En lo que respecta a Quintana Roo, el número de afiliados puede considerarse dentro de una cantidad regular para el mes de abril, aunque también se redujo la constancia de mantenerse, hasta marzo, cercano al límite de incrementos significativos.

A continuación, se presenta la contrastación de los resultados de cambios bajo criterios clásicos con respecto al estadístico $d$ de Cohen, como parte del metaanálisis.

Tabla 6. Nivel de cambios mediante delta de Cohen (metaanálisis).

\begin{tabular}{|c|l|c|c|c|c|}
\hline \multirow{2}{*}{ Meses } & \multicolumn{1}{|c|}{ Estado } & $\begin{array}{c}\text { Número de afiliados } \\
\text { (logaritmo natural) }\end{array}$ & Forma & Escala & $\begin{array}{c}\text { Delta de } \\
\text { Cohen }\end{array}$ \\
\hline \multirow{2}{*}{ Enero } & Aguascalientes & 12,73 & 12,25 & 0,2158 & $\mathbf{2 , 2 3}$ \\
\cline { 2 - 6 } & Quintana Roo & 13,07 & 12,41 & 0,3432 & $\mathbf{1 , 9 3}$ \\
\hline \multirow{2}{*}{ Febrero } & Aguascalientes & 12,74 & 12,26 & 0,2160 & $\mathbf{2 , 2 3}$ \\
\cline { 2 - 6 } & Quintana Roo & 13,07 & 12,42 & 0,3393 & $\mathbf{1 , 9 2}$ \\
\hline \multirow{2}{*}{ Marzo } & Aguascalientes & 12,73 & 12,27 & 0,2147 & $\mathbf{2 , 1 5}$ \\
\cline { 2 - 6 } & Quintana Roo & 12,96 & 12,43 & 0,3384 & $\mathbf{1 , 5 7}$ \\
\hline \multirow{2}{*}{ Abril } & Aguascalientes & 12,71 & 12,28 & 0,2133 & $\mathbf{2 , 0 2}$ \\
\cline { 2 - 6 } & Quintana Roo & 12,85 & 12,43 & 0,3388 & $\mathbf{1 , 2 4}$ \\
\hline
\end{tabular}

Fuente: Elaboración propia.

De acuerdo a los criterios adoptados para catalogar el nivel de cambios, en todos los meses del primer cuatrimestre de 2020 se presentaron grandes incrementos en el número de afiliados de Aguascalientes.

En lo que respecta a Quintana Roo, se presentaron incrementos en todos los períodos analizados, en todos los casos se presentaron cambios medios en el número de trabajadores afiliados.

\section{Conclusiones}

Uno de los factores económicos más afectados por la pandemia del COVID-19, ha sido sin lugar a duda el sector laboral, nadie podría negar la afectación a nivel nacional. Sin embargo, en agrupaciones mensuales con 22 observaciones por cada mes del año, los empleos formales registrados en el IMSS durante el primer cuatrimestre de 2020, no presentaron bajas alarmantes desde un punto de vista de la significancia estadística. Para el caso de los empleados en Aguascalientes, de comparar cifras anuales, como se realiza con el Producto Interno Bruto o la inflación, los empleos de enero, febrero, marzo y abril de 2020, fueron mayores a los registrados en esos mismos meses pero en el año anterior. En Quintana Roo sucedió una situación similar en los trabajos correspondientes a los meses de enero y febrero, no así en marzo y abril. A las empresas se les sugirió que no se despidiera personal ya que el Gobierno Federal se negó a emitir la declaratoria de "Emergencia Sanitaria", disfrazándola con la declaratoria "Contingencia Sanitaria", pues en caso de haber declarado la primera, solo obligaría a los patrones a cubrir un salario mínimo general hasta por un mes, acorde con el Artículo 429 de la Ley Federal del Trabajo. Sin embargo, al señalar esta pandemia de COVID-19 como "Contingencia sanitaria", permanecería la obligación del pago de salarios completos y de conservar la fuente de empleo.

Lo anterior explicaría la poca reducción, de acuerdo a la distribución de datos, de empleados formales en el primer cuatrimestre 2020. Sin embargo, pudo ser el aval para la existencia de convenios o acuerdos entre patrones y trabajadores para el goce anticipado de vacaciones o bien reducción de 
jornadas laborales o inclusive, ajuste al monto salarial argumentando cambios de categorías para poder mantener prestaciones formales, sobre todo las médicas y hospitalarias.

En los resultados para ambos estados se aprecia incremento del empleo formal en el periodo enero a abril de 2020, quizá contrario a resultados en otros estados del país. Tal situación estaría apalancada en el hecho de que muchas de las empresas en ambos estados son conglomerados de gran tamaño: en Quintana Roo grandes hoteles y operadoras turísticas involucradas, mientras que en Aguascalientes hay una gran cantidad de empresas del sector automotriz y de autopartes, y es probable que aunque no estén trabajando los empleados, se les haya conservado su plaza de trabajo en el IMSS, y aunque en algunos casos se dio una baja salarial por convenio con los empleados, como en el caso de Nissan Mexicana que pagó solo el 50\% de los salarios, aún así se conservaba el empleo hasta que se diera la reapertura, no dudando que esto se haya seguido por múltiples empresas del sector manufacturero y turístico, lo que ayudó a conservar el empleo.

Este estudio abre la interrogante para futuras líneas de investigación, con el mismo estudio en los meses subsecuentes para poder corroborar, si se continuó conservando la base del trabajo formal o bien hasta qué período fue posible conservar al menos, los registros de los empleados ante el IMSS.

\section{Referencias}

Aguilar, J. (2020). Resonancias. Instituto de Investigaciones Sociales de la UNAM. Desacargado de https://www.iis.unam.mx/blog/mexico-efectos-del-covid-19-en-el-mercado-del-trabajo/

Banco Mundial (2020). Empleo y desarrollo. Descargado de https://www.bancomundial.org/es/topic/jobsanddevelopment/overview

Cumming, G. (2013). Cohen's d needs to be readily interpretable: Comment on shieh. Behavior Research Methods, 45(4), 968-971.

De Jesús, L. \& Murillo, B. (2020). Efectos del Covid-19 en el empleo sectorial en México 2020. Economía Actual, 3, 23-29.

Dey, A.K. \& Kundu, D. (2009). Discriminating between the log-normal and log-logistic distributions. Communications in Statistics - Theory and Methods, 39(2), 280-292.

Gobierno de Quintana Roo (2020). Estrategia Reactivemos Quintana Roo dentro del plan Juntos Saldremos Adelante. Descargado de https://qroo.gob.mx/portal/estrategia-reactivemos-quintanaroo-dentro-del-plan-juntos-saldremos-adelante-conozcamos-en-que-consiste-y-como-funcionara/

Grace, A.W. \& Wood, I.A. (2012). Approximating the tail of the Anderson-Darling distribution. Computational Statistics and Data Analysis, 56(12), 4301-4311.

IMSS (2020). Trabajadores Asegurados - Series de Tiempo TABLEAU. Descargado de https://public.tableau.com/profile/imss.cpe\#!/vizhome/Histrico_4/Empleo_h?publish=yes

INEGI (2020). Ocupación - Población de 15 años y más según condición de actividad y disponibilidad, nacional trimestral. Descargado de https://www.inegi.org.mx/temas/empleo/default.html\#Tabulados

Negrete, R. (2011). El indicador de la polémica recurrente: la tasa de desocupación y el mercado laboral en México. Revista Internacional de Estadística y Geografia, 2(1), 22-43. 
OCDE (2020). Dirección de empleo, asuntos sociales y laborales. Descargado de https://mailchi.mp/oecd/imo-2018

Organización Internacional del Trabajo (2020). Panorama laboral en tiempos del COVID, México: OITAmericas.

Patiño, M. \& Cruz, G. (2020). Las medidas adoptadas por las entidades federativas ante la emergencia del Covid-19. Cuaderno de Investigación, 7, 1-162.

Presidencia de la República, 2020. DECRETO por el que se establecen las medidas de austeridad que deberán observar las dependencias y entidades de la Administración Pública Federal bajo los criterios que en el mismo se indican. México: Secretaría de Gobernación.

Rivero, E. (2020). Medidas para la recuperación económica ante la emergencia sanitaria Covid-19: Del crédito solidario al seguro de desempleo. Cuaderno de Investigación, 9, 1-57.

Shin, H., Jung, Y., Jeong, C., \& Heo, J. (2012). Assessment of modified Anderson-Darling test statistics for the generalized extreme value and generalized logistic distributions. Stochastic Environmental Research and Risk Assessment, 26(1), 105-114. 